\title{
VENUS CLOUD CONTRASTS
}

\author{
DAVID L. COFFEEN
}

Lunar and Planetary Lab., University of Arizona, Tucson, Ariz., U.S.A.

\begin{abstract}
Contrast of the Venus cloud patterns is measured by photoelectric scans using a sequence of narrow-band interference filters. The contrast appears constant in the range 3100-3600 $\AA$, drops to the limit of detectability at about $4100 \AA$, and is absent at longer wavelengths.
\end{abstract}

Photoelectric scans of Venus were made parallel to the terminator (Figure 1) using the two-channel scanner of Hall (1968), similar to the scans of Hall and Riley (1968). A $300 \mu(2.0$ arcsec $)$ circular focal plane aperture was used with a $1 \mathrm{sec}$ sweep time and a $6 \mathrm{~mm}$ sweep length. Eight Thin Films interference filters were used (Figure 2). The $\lambda 3557$ filter was placed permanently in Channel 1 to monitor the seeing. The other filters were placed successively in Channel 2. For each filter four scan integrations were made, each of $15 \mathrm{sec}$ duration. For two of these the Wollaston prism was set at approximately $45^{\circ}$ to the direction of polarization of Venus; for the other two the prism was turned by exactly $90^{\circ}$. Thus the averaged scans are insensitive to the presence of polarization.

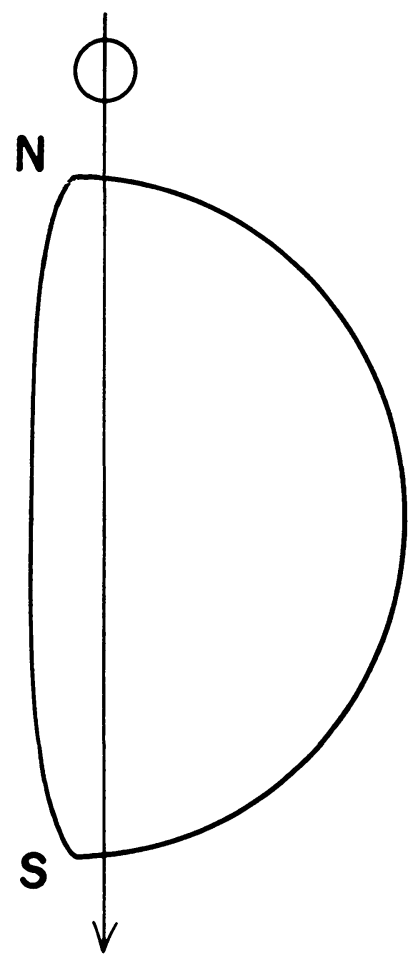

Fig. 1. Geometry for Venus scans at 83:0 phase angle. 


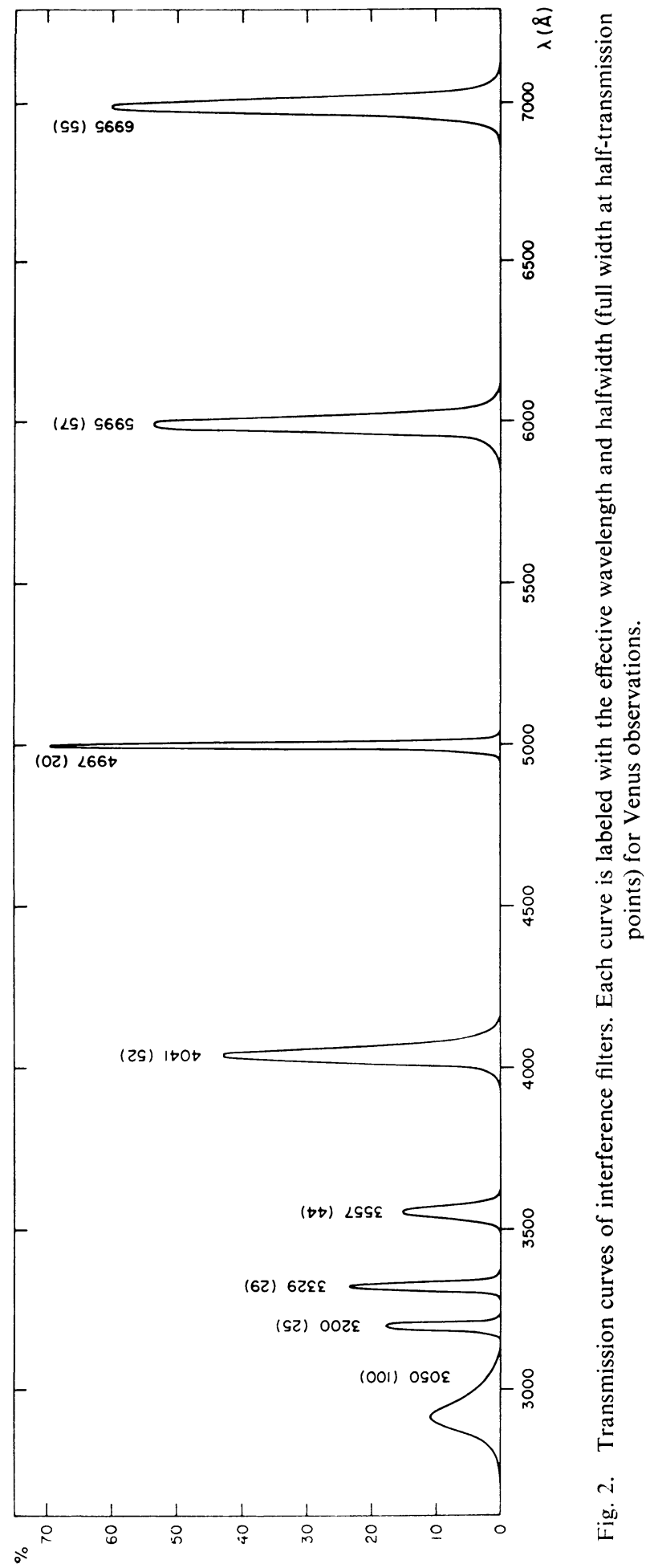



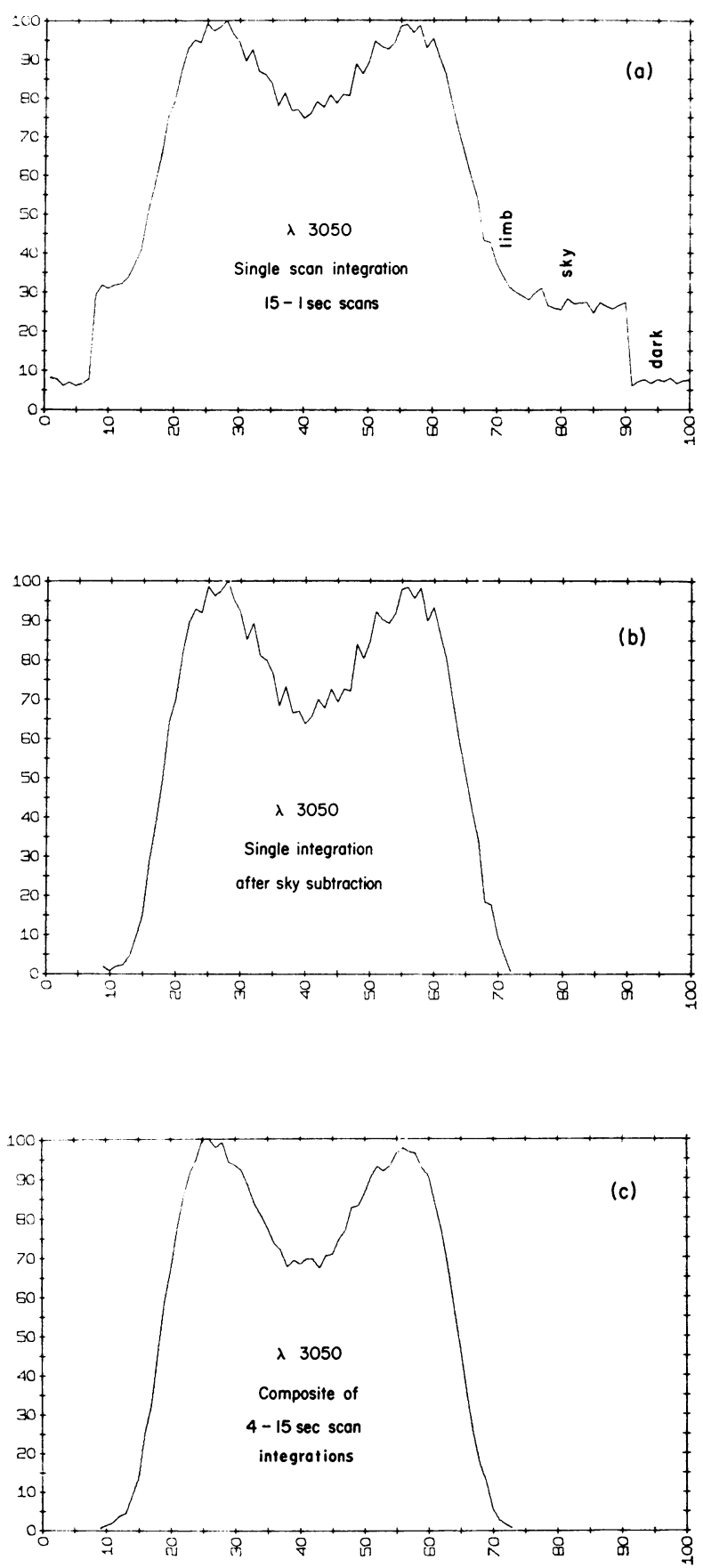

Fig. 3. Data reduction. The signal is somewhat noisy due to photon statistics. In spite of the large telescope diameter $\left(72^{\prime \prime}\right)$, the photon count is limited by the narrowness of the filters $(\Delta \lambda \sim 50 \AA)$, the focal plane aperture diameter $(300 \mu)$, and the limited scanning time. 


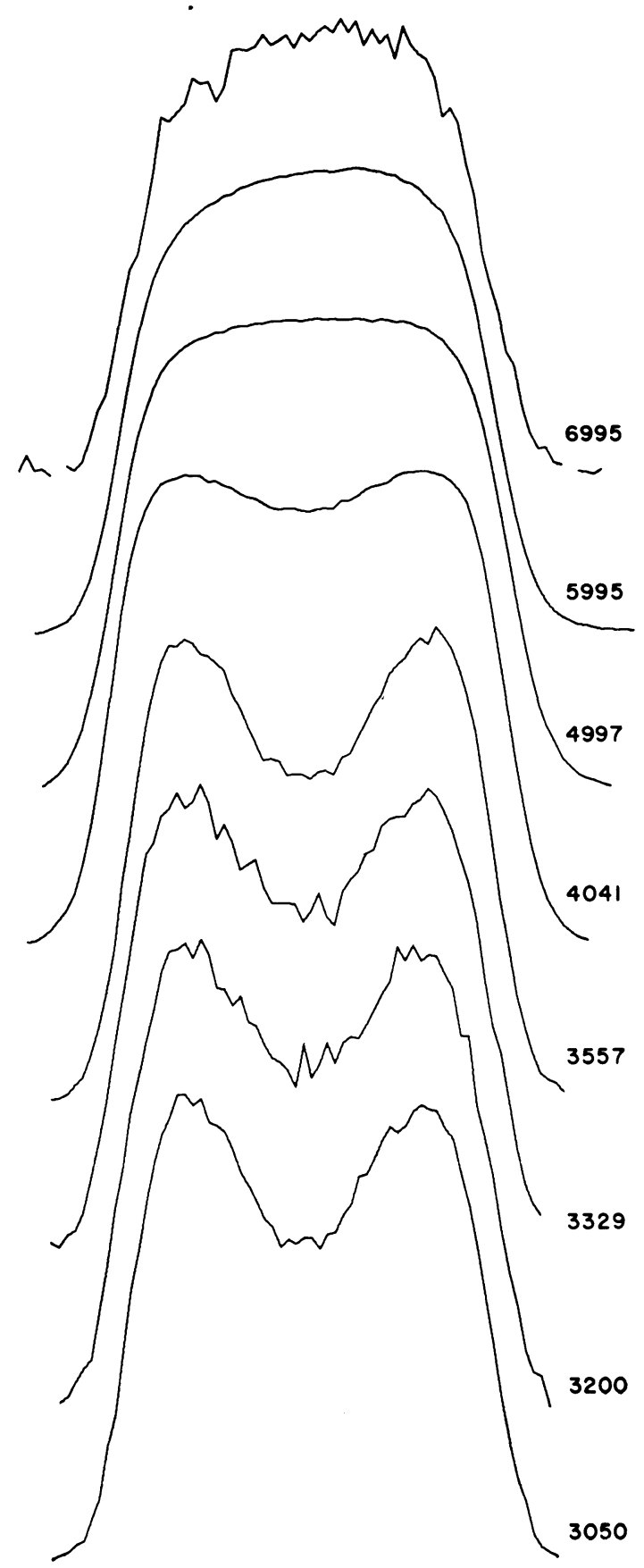

Fig. 4. Composite North-South scans of Venus at eight wavelengths. 
No depolarizer was used. The two photomultipliers were never interchanged, but the filters are sufficiently narrow that any spectral mismatch of the tubes is unimportant. One $16 / 1$ prescalar was used between the discriminator and multichannel analyzer for some of the filters to maintain an acceptable count rate. These daytime observations were made 2230-2400 UT, 17 January 1969 on the Perkins 72", at a phase angle of 83.0. Seeing excursions were 3-4 arcsec; the rms seeing was considerably better. Sky was clear but for a thin haze.

Figure 3 shows the steps in reduction of the scans made at one wavelength. The sky background varied from $0 \%$ to $76 \%$ of the total signal at the brightest region, depending on wavelength and time. The multichannel analyzer gives 100 points along the scan, or a resolution of about 0.4 arcsec. But the true resolution is determined by the seeing plus instrumental profiles. Figure 4 shows the final composite scans for all eight filters. On intercomparison of the scans there is no noticeable variation of seeing with wavelength. Signal to noise was particularly low at $\lambda 6995$ where the S-13 cathodes have low sensitivity.

Venus showed on this date a strong central dark region in the ultraviolet. The feature was observable visually through the $\lambda 4041$ filter in spite of the faintness of the image. The contrast will be defined as the intensity difference of the brightest and darkest regions, divided by the intensity of the brightest. Channel 1 was used to monitor the scan appearance at $\lambda 3557$ throughout the observing run. Changes in the seeing and/or in the positioning of the scan line caused small variations in the contrast measured on the sequence of composite Channel 1 scans, shown in the first column of Table I. The corresponding Channel 2 contrasts $C$ (column 3) were corrected for these

TABLE I

Cloud contrast $C=\left(I_{B}-I_{D}\right) / I_{B}$

\begin{tabular}{llll}
\hline Channel 1 & \multicolumn{2}{l}{ Channel 2} \\
\cline { 2 - 4 }$C(3557) \%$ & $\lambda$ & $C(\lambda) \%$ & $C^{\prime}(\lambda) \%$ \\
\hline- & & & 0 \\
30.0 & 6995 & 0 & 0 \\
29.5 & 5995 & 0 & 0 \\
31.5 & 4997 & 0 & 8.6 \\
28.0 & 4041 & 8.5 & 33.1 \\
29.0 & 3329 & 29.0 & 32.0 \\
32.0 & 3200 & 29.0 & 32.0 \\
\hline
\end{tabular}

variations by normalizing to the conditions of the $\lambda 3050$ scans. Thus for each filter:

$$
C^{\prime}(\lambda)=\frac{32.0 \%}{C(3557)} C(\lambda)
$$

The corrected values are plotted in Figure 5, showing the contrast as a function of wavelength for this date. For $\lambda 4997$ and longer wavelengths no central dip is seen, giving zero contrast. The central feature is detectable at $\lambda 4041$, and strengthens rapidly to $\lambda 3557$. The peak contrast of $32 \%$ is sensibly constant from $\lambda 3050$ to $\lambda 3557$. 


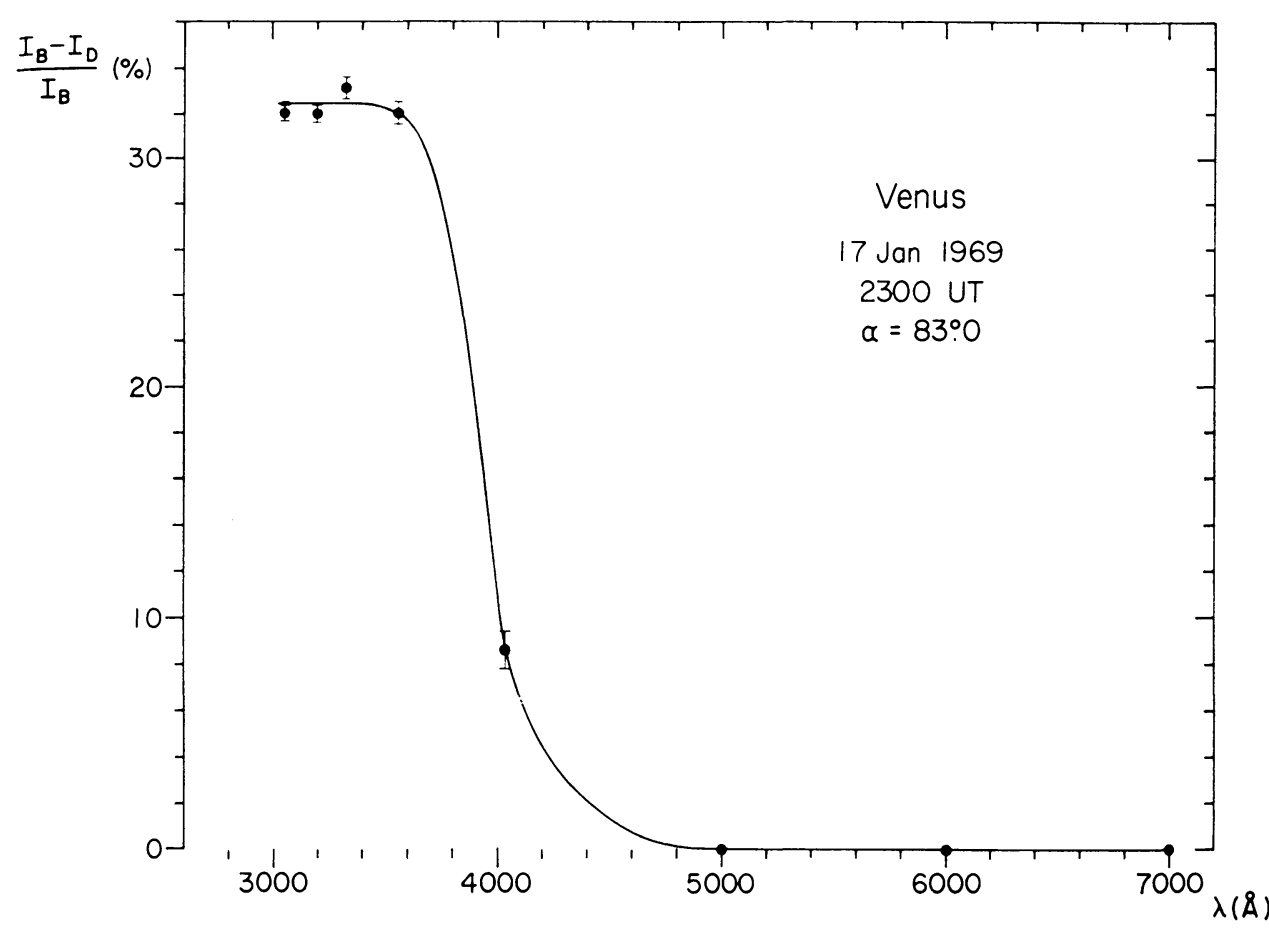

Fig. 5. Venus clouds as a function of wavelength. The measurements are taken from the scans of Figure 4, and are corrected slightly by the reference channel measurements as shown in Table I. The error bars represent one standard deviation from the mean of the four scan integrations made at each wavelength. The actual contrast values were taken from the composite scans of Figure 4. Those values could be read with a resolution of $0.5 \%$ contrast.
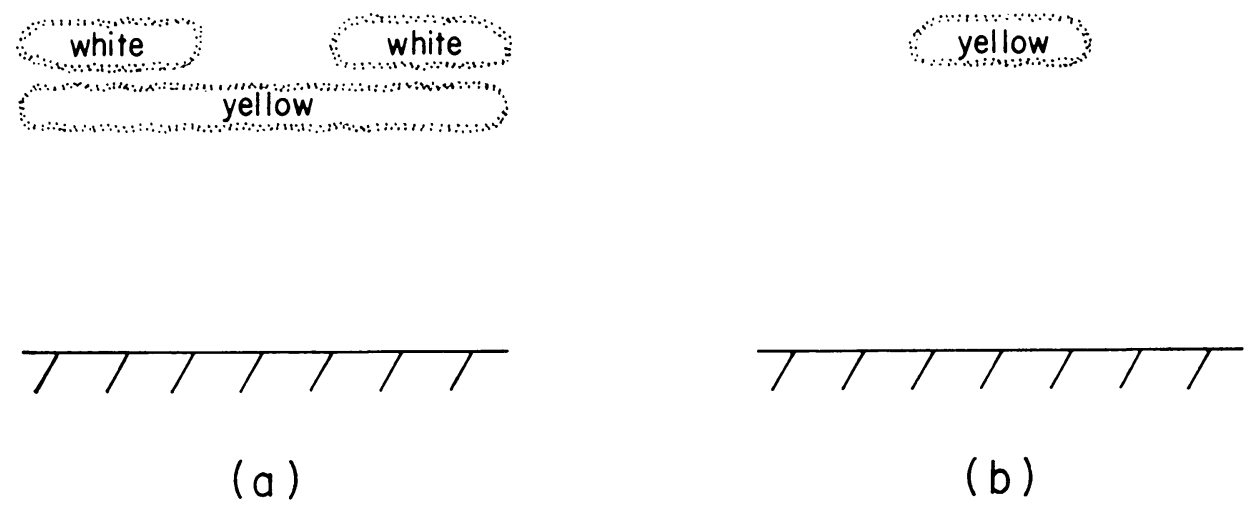

(a)

(b)

Fig. 6. Two possible explanations of the ultraviolet cloud contrasts. The central dark area is caused by absorbing clouds. The bright areas may be a white 'UV haze' cloud layer (a), or may simply be the light return from the deep molecular atmosphere (b). 
The relative smoothness of the contrast curve gives no suggestion of an origin as molecular absorption lines. The probable origin is absorption by the cloud particles. The total molecular optical depth of the atmosphere is $>100$ at $\lambda 3050$, assuming 100 atm surface pressure of pure $\mathrm{CO}_{2}$, giving a Bond albedo of $>97 \%$ (Kahle, 1968) at $\lambda 3050$, regardless of surface reflectivity. The cloud particles must be absorbing over a wide wavelength range, with more absorption at shorter wavelengths. This could occur with either one or two cloud layers, as shown in Figure 6.

Further observational work should be done on the absolute reflectivities of different cloud regions and on the wavelength dependence of the limb-darkening of Venus.

\section{Acknowledgment}

I wish to thank Dr. Hall for the use of the Lowell Observatory facilities.

\section{References}

Coffeen, D. L.: 1969, Astron. J. 74, 446.

Hall, J. S.: 1968, Lowell Observatory Bulletin 143, 7, 61.

Hall, J. S. and Riley, L. A.: 1968, Lowell Observatory Bulletin 145, 7, 83.

Kahle, A. B.: 1968, Astrophys. J. 151, 637. 